\title{
Las necesidades formativas de los nuevos comuni- cadores según los profesionales y su reflejo en los Grados de Comunicación
}

\author{
Montse VÁzQuez Gestal \\ Universidade de Vigo \\ mvgestal@uvigo.es \\ Ana Belén FERNÁNDEZ SOUTO
Universidade de Vigo
abfsouto@uvigo.es
}

Recibido: $19 / 07 / 2012$

Aceptado: 30/10/2012

\begin{abstract}
Resumen
La separación entre el mundo académico y el mundo profesional ha sido una afirmación constante en el ir y venir de las reformas educativas, sobre todo en el ámbito universitario, donde siempre se ha acusado a la academia de olvidarse del trabajo que van a desarrollar sus alumnos una vez abandonen las aulas en aras de una formación demasiado teórica. Ese intento de acercar uno y otro mundo se ha enarbolado como excusa en muchas ocasiones para reformar planes de estudio y no ha sido menos en la última reforma desarrollada al amparo del Espacio Europeo de Educación Superior. En esta tesitura desarrollamos esta investigación en la que decidimos acudir a profesionales diversos del mundo comunicativo para conocer cuál es la formación necesaria para desarrollar estos trabajos en la actualidad.
\end{abstract}

Palabras clave: grados, tendencias profesionales, formación, comunicación.

\section{The Training Needs of New Broadcasters according to Professionals and their Reflection in the Degrees of Communication}

\begin{abstract}
The separation between the academic world and the professional world has been a constant on the educational reforms, particularly at the University level, where has always accused the Academy to forget the work that will develop their students once abandon classrooms because of a too theoretical training. This attempt to bring one and another world has paraded as an excuse on many occasions to reform curricula and has not been less in the last reform developed under the umbrella of European space for higher education. In this frame of mind we develop this small research in which we decided to go to different communicative worldwide professionals to know about the training needed to develop these works today. Keywords: degrees, professional trends, training and communication.
\end{abstract}

\section{Referencia normalizada}

VÁZQUEZ GESTAL, Montse y FERNÁNDEZ SOUTO, Ana Belén (2012): "Las necesidades formativas de los nuevos comunicadores según los profesionales y su reflejo en los Grados de Comunicación". Estudios sobre el mensaje periodístico. Vol. 18, núm. especial noviembre, págs.: 889-897. Madrid, Servicio de Publicaciones de la Universidad Complutense.

Sumario: 1. Introducción. 2. La enseñanza de la comunicación. 3. Las necesidades formativas en las titulaciones de Publicidad y Relaciones Públicas. 4. Metodología. 5. Desarrollo: la opinión de los profesionales; 5.1. Publicidad; 5.2. Relaciones Públicas; 5.3. Fotógrafos; 5.4. Audiovisual. 6. Conclusiones. 7. Referencias bibliográficas. 


\section{Introducción}

El acercamiento entre el mundo profesional y académico ha sido una constante en la evolución de la enseñanza universitaria en España y la excusa para cambiar sistemas educativos con la intención de mejorar la formación de los futuros profesionales. Las universidades buscan afanosamente ofrecer a sus alumnos una formación lo más cercana posible a la realidad profesional. Una formación en la que los conocimientos se acerquen a lo que la calle y los profesionales demandan.

Durante muchos años, los expertos se han quejado de la falta de relación entre los conocimientos impartidos en las facultades y la realidad a la que los alumnos deben enfrentarse, una asignatura todavía pendiente en muchos casos, a pesar de que ese acercamiento a la realidad ha sido el argumento esgrimido para introducir "contenidos nuevos" en este último cambio universitario, conocido comúnmente como Plan Bolonia.

Un Plan nacido con la intención de unificar o acercar los conocimientos y la formación de la futura generación de trabajadores del Espacio Europeo, un intento más de construir una Europa en la que cambiar de lugar para estudiar y formarse no fuese un problema por la diferencia de planes y formatos académicos, sino una oportunidad de futuro. Sin embargo, parece que eso será difícil, cada país ha ido asimilado el concepto de Plan Bolonia de la manera que más le ha interesado, ya no solo en lo que respecta a los contenidos de los nuevos grados, sino incluso en cuanto a la denominación de los estudios o la duración de los mismos.

En el caso que nos ocupa, los estudios de Comunicación en España, esta diversificación no iba a ser una excepción. En el Libro Blanco de la Comunicación, en el propio Informe de la Comisión Evaluadora, se hace una llamada de atención sobre estos aspectos e indica que no se entiende por qué los trabajos de la Comisión encargada de los estudios de Comunicación se han desarrollado desde el principio atendiendo a una separación entre las tres titulaciones (Periodismo, Publicidad y Relaciones Públicas y Comunicación Audiovisual) cuando no presenta ni explica las diferencias entre ellas y no es capaz de justificar ni la necesidad de los tres tipos de grado, ni el desarrollo de los estudios en cuatro años, cuando en el resto de Europa se opta mayoritariamente por estudios de tres años y un cuarto de especialización, dentro de unos contenidos más genéricos desde el punto de vista comunicativo (Libro Blanco de la Comunicación: 13).

Es en este contexto donde nos situamos y situamos este artículo, intentando conocer si los nuevos grados permiten acercar al estudiante a la realidad profesional o si, por el contrario, le alejan un poco más de ella.

\section{La enseñanza de la comunicación}

Los estudios universitarios relacionados con el ámbito de la comunicación en España se desglosan en tres titulaciones: Periodismo, Publicidad y Relaciones Públicas y Comunicación Audiovisual.

La justificación para seguir manteniendo dicha separación, tal y como se recoge en el Informe de Trabajo desarrollado por la Comisión para el desarrollo del Libro Blanco, se apoya en la mayor especialización de las labores profesionales comunica- 
tivas, con una demanda de competencias y habilidades diferenciadas para cada una de las titulaciones (Libro Blanco de la Comunicación: 16 y ss)

Las tres titulaciones se imparten en nuestro país en más de 40 universidades, si bien es cierto que no todas cuentan con las tres titulaciones, y las tres comparten elementos comunes, lógicamente, pero también un grado de especialización que les ha permitido desarrollarse independientemente las unas de las otras, si bien es cierto que algunos perfiles y/o conocimientos se mezclan y entremezclan fuera y dentro de las aulas, fuera y dentro de los distintos trabajos. No resulta extraño encontrar licenciados en una u otra carrera ejerciendo labores que, en ocasiones, se considera deberían estar siendo desarrollados por profesionales de otra, pero la polivalencia ha sido también uno de los valores en alza en la formación actual, hablemos de la enseñanza que sea. Polivalencia destacada por muchos profesionales para quienes, tanto la situación de crisis actual como el progreso tecnológico, han hecho evolucionar los trabajos comunicativos de manera que hoy se les exige el desarrollo de labores que antes eran impensables.

A partir de la implantación del Plan Bolonia y de la aprobación de los distintos grados en Comunicación, teniendo en cuenta las directrices desarrolladas por el Libro Blanco, la distintas universidades y facultades fueron construyendo un catálogo de titulaciones supuestamente acorde a las necesidades demandadas por un entorno profesional cambiante, no solo desde el punto de vista de la formación, sino también por los cambios que el nuevo contexto ha provocado.

\section{Las necesidades formativas en las titulaciones en Publicidad y Relaciones Pú- blicas.}

Para el presente artículo hemos acotado, lógicamente, el espectro de estudio, por eso nos hemos centrado en las enseñanzas de Publicidad y Relaciones Públicas, concretamente en el grado de la Universidade de Vigo, cuya enseñanza se desarrolla en la Facultad de Ciencias Sociales y de la Comunicación, en el campus de Pontevedra.

Los grados se contruyen en torno a una serie de objetivos generales y específicos definidos en los Libros Blancos y que luego eran completados o particularizados por las comisiones encargadas del desarrollo de los grados en cada facultad/universidad. En el caso del grado que nos ocupa, dichos objetivos fueron asumidos integramente en la Memoria para el desarrollo del título, así como los perfiles profesionales establecidos en el Libro Blanco que determinan las enseñanzas a impartir y que, para Publicidad y Relaciones Públicas, son los siguientes:

- Gestores y estrategas publicitarios.

- Creativos.

- Directores de comunicación y relaciones públicas.

En el caso que nos ocupa, hablamos de un grado que comienza su andadura en el curso 2009/2010, por lo que su primera promoción saldrá al mercado laboral al finalizar el curso 2012-2013. 


\section{Metodología}

Nuestra hipótesis de partida era esa separación entre el mundo real y el mundo académico y nuestra intención era conocer si esa afirmación se reflejaba en el Grado en Publicidad y Relaciones Públicas de la Universidade de Vigo.

Por el lado profesional, necesitábamos conocer la opinión de los expertos sobre los conocimientos, habilidades y destrezas necesarias. A ellos que se les planteó las siguientes preguntas: qué conocimientos consideraban importantes teniendo en cuenta las tendencias y situación actual; qué formación que deberia ofrecer la Universidad y aquella otra complementaria que sería interesante para los estudiantes.

La información fue recopilada a lo largo de los meses de noviembre - diciembre de 2011 y abril - mayo de 2012. Los profesionales que respondieron a las preguntas planteadas fueron los siguientes:

- Inés Fernández. Estudio de Publicidad.

- Julinda Molares Cardoso. Anónimo Publicidad

- Verónica Barranco. Edelman

- Irene Fernández. Ekinocio comunicacion

- Cristóbal Fernández Muñoz. Director de Comunicación en Tuenti.

- Lorena Romero. Diario de Ferrol

- Teba Lorenzo. Directora de Ventas Yahoo Europa.

- Miguel Guardia Lorenzo. Freelance. Planner Digital

- Javier Magariños. Community Manager. Faro de Vigo.

- Angela Antón. Gestora en start up de compras de compras por Internet. www.duyudu.com

- Sandy Moragón. Social Media Strategist en Dnovae. Encargada de Marketing y Comunicación de Gafavintage.com.

- Eva Quintas Froufe. Documentalista y asistente de dirección en Tesauro, productora audiovisual.

- Eugenio Recuenco. Está considerado como uno de los fotógrafos creativos más importantes de España. Trabaja con Vogue España; ha publicado varios editoriales en la revista Madame Figaro. Ha trabajado tambien para Wad, Spoon, Vanity Fair, Stern, Zink y las españolas View, GQ, Hola. Ha realizado campañas para Custo Barcelona, Diesel, Puig, Mango, Caramelo, carrera y carrera, Florentino, Lodi, Chanel, Boucheron, Carolina herrera, Louis Vuitton España, Kina Fernández, Cimarron, Myrurgia o Caroche. Ver más en www.eugeniorecuenco.com

- Raul do Casar. Fotógrafo. Ha trabajado par marcas y revistas como Stradivarius, Burberry, Live, Grisby, Lois, Men's Health. Ha relaizado proyectos en colaboración con distintas agencias tales como Pink Orange, Emblema Comunicación y Sistema Diseño. Ver más en www.rauldocasar.com

- Gerardo García. Pionero como fotógrafo infantil en España, ha trabajado en los últimos años con familias desarrollando un estilo propio a través del uso de la luz natural y el entporno en cada uno de sus clientes. www.kidyart.com 


\section{Desarrollo: la opinión de los profesionales}

Una vez conocidas las opiniones, las hemos agrupado en bloques más o menos temáticos o áreas de trabajo de la siguiente forma:

\subsection{Publicidad}

Muchos cambios se han planteado en el ámbito publicitario en los últimos años, la sobresaturación y la evolución de un consumidor tremendamente activo, que ya no espera por el producto o la marca sino que va a buscarlo exigiéndole lo que le interesa, ha obligado a una mayor creatividad en las acciones publicitarias, pero también una mayor transparencia, naturalidad y humanidad. Para Inés Fernández (Agencia Estudio de Publicidad) se trata de "contar las cosas de manera diferente al resto [...] Además, hay que pensar on line y off line, ya no hay fronteras". El consumidor es inteligente ya no se compra con las ideas de siempre sino que exige nuevos formatos y nuevas razones para ser fiel. El consumidor es emisor y receptor, la idea de participación, colectividad y grupo de las redes sociales le fascina. Quiere participar y a ello se deben apuntar las marcas, como por ejemplo hace Freixenet, que este año para su campaña de Navidad abre un concurso para que participen todos los que tengan razones para hacer un brindis, subiendo sus videos, ideas... de entre los que se elegirán aquellos con los que se construirá el spot navideño de este año, dirigido por Bigas Luna (www.youtube.com/freixenet).

Esta situación obliga a los profesionales a estar al día, mirar alrededor, leer mucho y de todo. Aprender a escribir, a redactar. Culturizarse: todo interesa: ver publicidad y televisión, cine, salir a la calle, ir al teatro, leer novelas, navegar por Internet, jugar a videojuegos, relacionarse dentro y fuera de la red... Todo sirve para formarse. Saber expresarse. Julinda Molares (Agencia de publicidad Anónimo) destaca la necesidad del apoyo teórico para buscar ideas, "debe existir una educación basada en establecer conexiones neuronales que le permitan (al profesional) pensar diferente a todas horas". Una cuestión obvia, que ya en su día profesionales clásicos, como Young (1982), o más actuales, como Furones (2010)1, enarbolaron como la base de la creatividad pero que hoy en día es reivindicada más que nunca por los profesionales. Ello obliga a pensar en la necesidad de materias de arte, literatura, cine... como lugar para encontrar nuevas conexiones, ideas o inspiraciones para atraer a un público que cada vez se sorprende menos pero que está deseoso de hacerlo. Es imprescindible conocer las herramientas y los programas informáticos, programas de diseño gráfico, programas de edición y $3 \mathrm{D}$, pero también las técnicas para la producción de ideas y la curiosidad para observar y asimilar todo lo que ocurre a nuestro alrededor.

Desde Ekinocio Comunicación se plantea la necesidad de cambiar de registro debido a la aparición de los nuevos medios y las demandas por parte de los anunciantes

${ }^{1}$ Tanto en sus libros como en sus artículos, cualquiera de estos autores destaca esa necesidad del permanente contacto con todo lo que ocurre alrededor, así como el conocimiento de disciplinas que permitan encontrar referencias que el consumidor identifica y nos permita acercarle la marca o el producto, ya sean referencias cinematográficas, artísticas, literarias o familiares. 
de formar parte de la web, lo que significa adaptar los conocimientos teóricos a los proyectos que se presentan para los distintos medios y eso significa que en la formación de los publicitarios no puede faltar la lectura profesional y genérica, la importancia de saber usar el lenguaje correctamente y de expresarse de forma adecuada, conocer el lenguaje de los medios, pero también el del anunciante. Destacan además que, en los pequeños estudios, todos trabajan a la vez en el proyecto, no hay tanta división en departamentos estanco como en las grandes agencias, lo que obliga a saber relacionarse con el cliente directamente y, a la vez, saber presentarle soluciones al problema que plantea.

\subsection{Relaciones Públicas}

En este sector la necesidad de desarrollar una comunicación sólida y sostenida en el tiempo para que una marca sea relevante, es un tema vital. Verónica Barranco (Agencia de Relaciones Públicas Edelman Spain) afirma que "para que una marca sea relevante ya no le basta ceñirse a los canales actuales [...], sino que tiene que establecer una comunicación sólida y sostenida en el tiempo, un diálogo multidireccional con otros públicos implicados". En la situación actual, la confianza se sitúa como uno de los valores más importantes, y eso ha obligado a las marcas a buscar fuentes informativas que le ayuden a crear esa confianza y compromiso con sus usuarios y/o consumidores, lo que actualmente se denomina "engagement".

$\mathrm{Y}$ ese compromiso entre marca y usuario lleva a desarrollar campañas como la construida por Tous a través de Twiter e Internet: el objetivo de la marca era llegar a los 18403 seguidores en Twiter, a través del hashtag \#1843lovers, cuando esto ocurrió el número de transformó en "I love You", agradeciendo a sus seguidores la acción con un regalo personalizado2. "Esta acción no estaba pensada para ganar seguidores sino para fidelizar", declaraba el director del área digital de Tous, Xavier Colomé. Eso es lo que buscan las marcas, crear conversaciones y experiencias, fidelizar a un consumidor que cada vez exige más de la marca que consume. La web 2.0 ha dado voz al consumidor y las empresas y marcas deben responderle, volcándose en crear historias, contenidos, que sean relevantes para él. Y ese compromiso se extiende dentro de la propia compañía, ya que los primeros embajadores de la marca son los propios empleados, son una de las fuentes más confiables que la empresa debe cuidar, pues son los primeros que crean imagen.

Y para desarrollar estas actividades, los idiomas y la redacción o el saber expresarse en los nuevos medios vuelven a configurarse como los conocimientos más importantes en la formación de los alumnos, destacándose en el ámbito de los profesionales de las Relaciones Públicas, así como conocer y comprender mejor los entornos empresariales, donde el buen uso de la comunicación cada vez es más necesario. La importancia de las redes sociales y su aplicación a este ámbito es destacado por Cristóbal Fernández (Director de Comunicación en Tuenti), pues las nuevas herramientas dan lugar a nuevas metodologías de trabajo que es preciso conocer, como

2 En la página www.18403lovers.com se puede ver un mosaico con todas las piezas que Tous ha regalado a los participantes en la acción y que todavía continúa abierta. 
"la medición de los resultados de programas de las relaciones públicas", una tendencia de futuro muy necesaria en estos momentos de crisis y recortes en los presupuestos de comunicación, y sobre la que deberían introducirse conocimientos teórico-prácticos en las facultades.

En un terreno más local, el desconocimiento en las instituciones pequeñas del trabajo de los profesionales de la comunicación, la ausencia de trabajo con gabinetes de comunicación o relaciones públicas, lo que conlleva errores graves en la organización de eventos a pequeña o mediana escala, es la principal cuestión a rectificar aunque, tal vez el mayor problema es que ni siquiera las entidades pequeñas, caso de ayuntamientos, son concientes de que ello es un problema.

Los representantes de este sector destacan lo que ya nos advertían los profesionales de la publicidad: el conocimiento de los idiomas, imprescindible en los tiempos que corren, saber redactar, un mayor conocimiento de los entornos empresariales y la formación en el ámbito digital pero también en el de la investigación. Lorena Romero (Diario de Ferrol) incide en saber trabajar con programas informáticos enfocados al ámbito comunicativo, así como conocer conceptos como comunicación interna y externa o cuestiones de protocolo institucional.

\subsection{Fotógrafos}

Para Raúl do Casar, "en la universidad se enseñan muchas cosas y se aprenden pocas", por eso debería centrarse en asentar unas bases útiles que luego el alumno pueda explotar y desarrollar. Potenciar la motivación, orientación y optimización de conocimientos. Para Do Casar es precisa una formación especializada que no solo se desarrolla a través de las prácticas sino que el alumno debe asistir también a cursos impartidos por profesionales. Es imprescindible conocer disciplinas como la literatura, el cine, la historia... Conocer a los grandes de la fotografía, saber identificar trabajos de calidad y tener criterio, tal y cómo afirma Gerardo García "al fin y al cabo el resultado del trabajo de un fotógrafo es consecuencia de todo lo que ha visto, leído, viajado, experimentado...".

Las tendencias en el sector fotográfico se adecúan y varían a medida que la sociedad y el individuo evolucionan por ello el alumno debe conocer e identificar los que ocurre a su alrededor y los cambios que eso conlleva.

En un ámbito más especializado aún, caso de la moda, Do Casar destaca actualmente una búsqueda de la sencillez "con fondos totalmente neutros y modelos naturales". Simplicidad y más simplicidad. Gerardo García destaca la facilidad de acceso al mundo de la fotografía, equipos mejores y más baratos, la posibilidad de acceder a clientes en todo el mundo, si bien la desventaja es que, más que nunca, debes encontrar un hueco, ofrecer algo realmente diferente y único a los clientes, ello ofrece unas posibilidades a los estudiantes que deben saber aprovechar, no solo esperando que les enseñen, sino también volando ellos a partir de las pautas que conocen. Para Eugenio Recuenco toda la fotografía comercial "está derivando hacia un mundo en movimiento", un momento de prueba y adaptación donde cada vez se requiere más material en video.

Es importante conocer la parte de negocio que tiene la fotografía, hoy más que nunca con los recursos, que existen uno debe saber moverse. Pero además, el fotógrafo 
tiene que saber contar historias, y poseer conocimientos de realización en movimiento, color, pose, concepto...

\subsection{Audiovisual}

En este ámbito hemos incluido la opinión de profesionales diversos, intentando integrar en un paraguas bastante amplio las opiniones sobre ocupaciones que entremezclan en ocasiones contenidos y soportes. Aquí incluimos la opinión de profesionales que trabajan en productoras audiovisuales, marketing on line, gestores de contenidos web, agencias de compras en Internet...

Los perfiles demandados están muy centrados en el ámbito on line: community manager, especialistas en SEM/SEO y especialistas en comercio electrónico. Comunicación digital y nuevas formas de comunicación en Internet. Pero además, la tendencia se orienta también la optimización de recursos en todos los sentidos, por ejemplo, tal y como destaca Eva Quintas (documentalista y asistente de dirección en Tesauro, productora audiovisual), "se demandan conocimientos y experiencia práctica en edición, maquetación, diseño, guión y dirección para optimizar al máximo las funciones de cada profesional" que cumplan un amplio rango de funciones, no siempre relacionadas entre si.

La tendencia es actualizarse o morir, Internet y más Internet, como señala Miguel Guardia (freelance, Planner Digital), y la actuación del consumidor ante la fragmentación de los medios. Conocer a los usuarios y cómo se comportan, sobre todo por la velocidad en los cambios y su mayor exigencia desde todas las perspectivas; como señala Angela Antón (gestora en start up de compras por Internet. www.duyudu.com) "hay que ponerse al día para poder trabajar, lograr interactuar con los potenciales usuarios [...] los cambios son constantes y tal vez no estemos lo suficientemente preparados". Hay que ofrecer contenidos interesantes, potenciar la interacción, buscar la empatía con el usuario como también destaca Javier Magariños (Faro de Vigo).

Todos estos cambios obligan a trabajar con plataformas tecnológicas que gestionan contenidos on line, sistemas de monitorización de campañas publicitarias, programas de análisis de audiencias y tráfico web, cuestión en la que hace especial hincapié Teba Lorenzo (directora de ventas Yahoo Europa).

La falta de formación en el ámbito on line es destacado, entre otros, por Sandy Moragón (Social Media Strategist en Dnovae; encargada de Marketing y Comunicación de Gafavintage.com) teniendo en cuenta que, en este momento es, tal vez, el sector que más posibilidades de trabajo está ofreciendo. Junto a esto vuelven a incidir los profesionales en la necesidad de los idiomas como algo que está ya fuera de toda duda.

\section{Conclusiones}

A la vista de las opiniones profesionales destacamos una serie de conclusiones, una vez contrapuesta dicha información con el plan de estudios observado:

Los profesionales destacan la necesidad de saber contar historias, de buscar ideas, de producir resultados novedosos y originales. Sin embargo, apenas hay materias en este grado que tengan en cuenta estos contenidos, salvo las que tienen que ver con el 
ámbito de la creatividad y éstas no han aumentado con respecto a los planes de estudio anteriores.

A pesar de la reconocida importancia de conocer el entorno digital, se sigue aludiendo a los medios tradicionales en la mayoría de las materias, si bien es cierto que hay una asignatura optativa en el cuarto curso sobre nuevos medios y soportes.

Sobre el conocimiento del consumidor, sus relaciones con la tecnología, los medios, sus cambios en actitudes, los modos de entender la vida, de relacionarse con los demás, no encontramos demasiados contenidos que tengan en cuenta este tipo de cuestiones, solamente una materia que imparte el área de Sociología (Estructura social, consumo y estilos de vida) que no tiene continuidad en el grado.

Tampoco encontramos materias que ayuden al alumno a acercarse al consumidor, a conocer tendencias o referencias, identificarlas y aplicarlas al margen del medio que se fuese a utilizar. Conocimientos como arte, historia, literatura, que enseñen a buscar referentes y modos de conexión con el consumidor, han desaparecido del nuevo plan de estudios.

Los idiomas son otra reivindicación que sigue sin tener respuesta desde el mundo académico.

Teniendo en cuenta lo observado, podemos decir que, en el caso que nos ocupa, se sigue manteniendo esa brecha entre el ámbito profesional y el académico. Bien es cierto que algunos aspectos han mejorado con respecto al plan de estudios anterior. Sin embargo, siguen siendo muchas las carencias encontradas una vez escuchados a los profesionales

\section{Referencias bibliográficas}

FURONES, Miguel Ángel (2010): Tres mil años de Internet. Madrid, Publicaciones Profesionales, S.L.

Libro Blanco. Títulos de Grado en Comunicación (2005). Madrid, Agencia Nacional para la Evaluación de la Calidad (ANECA)

MARKETINGNEWS.es: "Tous un buen ejemplo de engagement". En http://www.marketingnews.es/bienes-duraderos/noticia/1069656028105/touspremia-seguidores-twitter-joya-virtual.1.html. Publicado y consultado el 18 de octubre de 2012.

MEMORIA para la solicitud de verificación del Título del 30 de octubre de 2007. Publicado en BOE el 26 de septiembre de 2008.

YOUNG, James Webb (1982): Una técnica para producir ideas. Madrid, Eresma.

Montse VÁZQUEZ GESTAL

Universidade de Vigo

mvgestal@uvigo.es

\section{Ana Belén FERNÁNDEZ SOUTO}

Universidade de Vigo

abfsouto@uvigo.es 